\title{
Cultivated sunflower high-throughput genotyping and lipidomic profiling
}

\author{
Chernova A. ${ }^{*}$, Singh A. ${ }^{2}$, Sherbina K. ${ }^{2}$, Chang P. ${ }^{2}$, Mazin P. ${ }^{1,3}$, Gubaev R. ${ }^{1}$, \\ Goryunova S..$^{1,4}$, Goryunov D. ${ }^{1,5}$, Boldyrev S. ${ }^{1}$, Vanushkina A. ${ }^{1}$, Anikanov N. ${ }^{1}$, \\ Yushina E. ${ }^{1,6}$, Martynova E. ${ }^{1}$, Demurin Y. ${ }^{7}$, Mukhina Z. ${ }^{8}$, Gavrilova V. ${ }^{9}$, Anisimova I. ${ }^{9}$, \\ Karabitsina Y. ${ }^{9}$, Nuzhdin S. ${ }^{2}$, Khaitovich P. ${ }^{1}$ \\ ${ }^{1}$ Skolkovo Institute of Science and Technology, Moscow, Russia \\ ${ }^{2}$ University of Southern California, Los Angeles, CA, USA \\ ${ }^{3}$ Institute for Information Transmission Problems, RAS, Moscow, Russia \\ ${ }^{4}$ Vavilov Institute of General Genetics, RAS, Moscow, Russia \\ ${ }^{5}$ Belozersky Institute of Physico-Chemical Biology, MSU, Moscow, Russia \\ ${ }^{6}$ Pirogov Russian National Research Medical Institute, Moscow, Russia \\ ${ }^{7}$ Pustovoit All-Russian Research Institute of Oil Crops, Krasnodar, Russia \\ ${ }^{8}$ All-Russian Rice Research Institute, Krasnodar, Russia \\ ${ }^{9}$ N.I. Vavilov All-Russian Research Institute of Plant Genetic Resources (VIR), St. Petersburg, Russia \\ *e-mail: alina.chernova@gmail.com
}

Cultivated sunflower in one of key plants used by human. It is an important oilseed crop that was domesticated from the wild sunflower approximately 4000 years ago. Now sunflower is mainly planted for the seed oil. Selection of hybrids with changed oil properties is one of the basic directions in oilseed crops hybrid breeding. Full sunflower genome assembly released by Badouin et al. made good possibilities for large-scale genome wide association studies (GWAS) which result can be implemented in sunflower genomic selection and help to speed up breeding programs. In this study we perform high-throughput genotyping (GBS-sequencing) and lipidomic phenotyping on 600 inbred sunflower lines. For lipid profiling we use ultra high performance liquid chromatography coupled with mass-spectrometry (UPLC-MS). UPLC-MS is a very powerful tool for lipidomics, which allow simultaneous profiling of several hundreds different lipids extracted from a single plant sample. Here we perform comparative lipidomic study in sunflower and combine NGS based genotyping with high performance phenotyping technology and show advantages of this approach for agricultural proposes. We have identified 2360111 SNPs and 1000 lipid molecules. GWAS were performed. Significant associations between molecular phenotypes and SNPs were identified. Our results extend current knowledge of sunflower metabolism and give new insights on development of approaches in oil-seed crop genomic selection. 